\section{Cellular Physiology} and Biochemistry Published online: October 30, 2015

Accepted: September 21, 2015

\title{
MiR-429 Induces Gastric Carcinoma Cell Apoptosis Through Bcl-2
}

\author{
Ping Zhu Jingping Zhang Jianfei Zhu Jun Shi Qiuwei Zhu Yuanyuan Gao \\ Department of General Surgery, Changzhou NO.2 People's Hospital, Changzhou, China
}

\section{Key Words}

Gastric carcinoma (GC) • Bcl-2 • MiR-429 • Chemotherapy

\begin{abstract}
Background/Aims: MicroRNAs (miRNAs) play an essential role in the tumorigenesis of gastric carcinoma (GC). MiR-429 has been recently reported to inhibit GC growth, but the underlying mechanisms are not clear. Methods: Here, we studied the levels of miR-429 and anti-apoptotic protein $\mathrm{BCl}-2$ in GC specimens. We performed bioinformatics analyses and used luciferasereporter assay to analyze the relationship between miR-429 and Bcl-2 in GC cells. Cell survival upon Fluorouracil treatment was analyzed in a CCK assay. Cell apoptosis was measured by flow cytometry based FITC Annexin V apoptosis detection assay. Results: MiR-429 levels were significantly decreased and $\mathrm{BCl}-2$ levels were significantly increased in GC specimens, compared to the paired adjacent non-tumor gastric tissue. Moreover, the levels of miR-429 and $\mathrm{Bcl}-2$ inversely correlated in GC specimens. MiR-429-low subjects had an overall inferior survival, compared to miR-429-high subjects. Bioinformatics analyses showed that miR-429 targeted the 3'-UTR of Bcl-2 mRNA to inhibit its translation, which was confirmed by luciferase-reporter assay. Overexpression of miR-429 inhibited $\mathrm{Bcl}-2$-mediated cell survival against apoptosis induced by Fluorouracil, while depletion of miR-429 augmented it. Conclusion: Our data suggest that miR-429 suppression in GC promotes Bcl-2-mediated cancer cell survival against chemotherapy-induced cell death. Re-expression of miR-429 levels in GC cells may enhance cancer apoptosis during chemotherapy.
\end{abstract}

\section{Introduction}

Gastric carcinoma (GC) causes the highest cases of cancer-related death in China [14]. Chemotherapy, e.g. Fluorouracil (5-FU) treatment, has been used as a supplementary treatment to surgical removal of the primary cancer, which improves the survival of the patients [5-9]. However, some GCs have been shown to be resistant to 5-FU treatment and the molecular mechanisms underlying this phenomenon are not completely understood. The resistance of a specific cancer to a specific chemotherapy may result from enhanced anti-apoptotic potentials of the cancer cells in a particular setting [10-12]. Cellular apoptosis

Ping Zhu

KARGER 125
Department of General Surgery, Changzhou NO.2 People's Hospital, 29 Xing Long Xiang Road, Changzhou 213003, Jiangsu Province, (China) Tel. +8651988113676, Fax +8651988115560, E-Mail zhuping_15@163.com 
is regulated by apoptosis activating proteins, e.g. Bid, Bak, Bad, and apoptosis suppressors, e.g. Bcl-2 [13-19].

Great efforts have been made to understand the molecular carcinogenesis of GC, as well as to search an effective molecular therapy to assist the surgery and chemotherapy [20]. Growing evidence has suggested that aberrant expression of MicroRNAs (miRNAs) contributes to GC initiation, progression, outgrowth, metastases and resistance to chemotherapy [2, 21, 22]. MiRNA is a class of non-coding small RNA of comprised of about 18-23 nucleotides, and regulate the gene expression at protein level, through their basepairing with the $3^{\prime}$-untranslated region $\left(3^{\prime}\right.$-UTR) of the mRNA of the target gene $[23,24]$. Protein levels are controlled by gene expression, protein translation and protein degradation. Thus, miRNAs play a critical role in regulating intracellular protein levels in many biological events including development of a tumor [2, 21, 22, 25]. However, miR-429 is a rarely studied miRNA, and its involvement in the carcinogenesis has not been reported.

Here, we showed that miR-429 levels were significantly decreased and Bcl-2 levels were significantly increased in GC specimens, compared to the paired adjacent non-tumor gastric tissue. Moreover, the levels of miR-429 and Bcl-2 inversely correlated in GC specimens. MiR-429-low subjects had an overall inferior survival, compared to miR-429-high subjects. Bioinformatics analyses showed that miR-429 targeted the 3'-UTR of Bcl-2 mRNA to inhibit its translation, which was confirmed by luciferase-reporter assay. Overexpression of miR429 inhibited Bcl-2-mediated cell survival against apoptosis induced by Fluorouracil, while depletion of miR-429 augmented it. Together, our data suggest that miR-429 suppression in GC promotes Bcl-2-mediated cancer cell survival against chemotherapy-induced cell death. Re-expression of miR-429 levels in GC cells may enhance cancer apoptosis during chemotherapy.

\section{Materials and Methods}

\section{Patient tissue specimens}

Forty resected GC specimens (paired GC and the adjacent non-tumor gastric tissue (NT)) in this study were histologically and clinically diagnosed at the Changzhou NO.2 People's Hospital from 2010 to 2014. For the use of these clinical materials for research purposes, prior patient's consents and approval from the Institutional Research Ethics Committee were obtained.

\section{Cell line and reagents}

A human GC cell line AGS was purchased from American Type Culture Collection (ATCC, Rockville, MD, USA), and was cultured in RPMI1640 medium (Invitrogen, Carlsbad, CA, USA) supplemented with 15\% fetal bovine serum (FBS; Sigma-Aldrich, St Louis, MO, USA) in a humidified chamber with $5 \% \mathrm{CO}_{2}$ at $37^{\circ} \mathrm{C}$. Fluorouracil (5-FU; Sigma-Aldrich) was prepared in a stock of $1 \mathrm{mmol} / \mathrm{l}$ and applied to the cultured GC cells at $1 \mu \mathrm{mol} / \mathrm{l}, 5 \mu \mathrm{mol} / \mathrm{l}$ and $25 \mu \mathrm{mol} / \mathrm{l}$, respectively.

\section{Plasmid transfection}

MiR-429-expressing and antisense (as) plasmids were all purchased (RiboBio Co., Ltd., Guangzhou, Guangdong, China). Transfection was performed with Lipofectamine 2000 reagent (Invitrogen) using 1 $\mu \mathrm{g}$ plasmids, according to the instructions of the manufacturer. The cells that were transfected with null plasmid as a control (null). Transfected cells expressing miR-429, or as-miR-429, or control null were purified by flow cytometry based on GFP.

Western blot

The protein was extracted from the GC or NT specimens, or from cultured cells, in RIPA lysis buffer (1\% NP40, 0.1\% SDS, $100 \mu \mathrm{g} / \mathrm{ml}$ phenylmethylsulfonyl fluoride, $0.5 \%$ sodium deoxycholate, in PBS) on ice. The supernatants were collected after centrifugation at $12000 \times \mathrm{g}$ at $4^{\circ} \mathrm{C}$ for $20 \mathrm{~min}$. Protein concentration was determined using a BCA protein assay kit (Bio-rad, China), and whole lysates were mixed with $4 \times$ SDS 


\section{Cellular Physiology Cell Physiol Biochem 2015;37:1572-1580

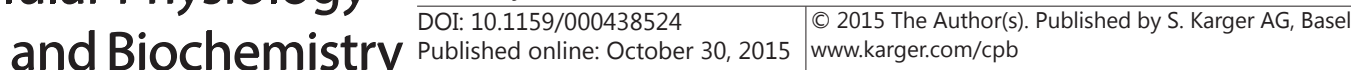 \\ Zhu et al.: MiR-429 Induces GC Cell Apoptosis via Bcl-2}

loading buffer $(125 \mathrm{mmol} / \mathrm{l}$ Tris-HCl, $4 \%$ SDS, $20 \%$ glycerol, $100 \mathrm{mmol} / \mathrm{l}$ DTT, and $0.2 \%$ bromophenol blue) at a ratio of $1: 3$. Samples were heated at $100^{\circ} \mathrm{C}$ for $5 \mathrm{~min}$ and were separated on SDS-polyacrylamide gels. The separated proteins were then transferred to a PVDF membrane. The membrane blots were first probed with a primary antibody. After incubation with horseradish peroxidase-conjugated second antibody, autoradiograms were prepared using the enhanced chemiluminescent system to visualize the protein antigen. The signals were recorded using X-ray film. Primary antibodies were rabbit anti-Bcl-2 and anti$\alpha$-tubulin (Cell Signaling, San Jose, CA, USA). Secondary antibody is HRP-conjugated anti-rabbit (Jackson ImmunoResearch Labs, West Grove, PA, USA). Blotting images were representative from 5 repeats. $\alpha$-tubulin was used as a protein loading control. The protein levels were first normalized to $\alpha$-tubulin, and then normalized to the experimental controls. Densitometry of Western blots was quantified with NIH ImageJ software (Bethesda, MD, USA).

\section{Quantitative RT-PCR}

Total RNA and miRNAs were extracted from tissue specimens or from cultured cells with miRNeasy mini kit or RNeasy kit (Qiagen, Hilden, Germany), respectively. Complementary DNA (cDNA) was randomly primed from $2 \mu \mathrm{g}$ of total RNA using High-Capacity cDNA Reverse Transcription Kit (Applied Biosystems, Foster City, CA, USA). RT-qPCR was subsequently performed in triplicate with QuantiTect SYBR Green PCR Kit (Qiagen). All primers were purchased from Qiagen. Data were collected and analyzed using $2^{-\Delta \Delta C t}$ method for quantification of the relative mRNA expression levels. Values of genes were first normalized against $\alpha$-tubulin, and then compared to the experimental controls.

MiRNA target prediction and 3'-UTR luciferase-reporter assay

MiRNAs targets were predicted as has been described before, using the algorithms TargetSan (https:// www.targetscan.org) [26]. Luciferase-reporters were successfully constructed using molecular cloning technology. Target sequences for Bcl-2 miRNA 3'-UTR clone and a site mutation were purchased from Creative Biogene (Shirley, NY, USA). MiR-429-modified GC cells were seeded in 24-well plates for 24 hours, after which they were transfected with $1 \mu$ g of Luciferase-reporter plasmids per well. Luciferase activities were measured using the dual-luciferase reporter gene assay kit (Promega, Beijing, China), according to the manufacturer's instructions.

Cell viability by cell counting kit-8 (CCK-8) assay

The CCK-8 detection kit (Sigma-Aldrich) was used to measure cell viability according to the manufacturer's instructions. Briefly, cells were seeded in a 96-well microplate at a density of $5 \times 10^{4} / \mathrm{ml}$. After $24 \mathrm{~h}$, cells were treated with resveratrol. Subsequently, CCK- 8 solution $(20 \mathrm{ml} /$ well) was added and the plate was incubated at $37^{\circ} \mathrm{C}$ for 2 hours. The viable cells were counted by absorbance measurements with a monochromator microplate reader at a wavelength of $450 \mathrm{~nm}$. The optical density value was reported as the percentage of cell viability in relation to the control group (set as $100 \%$ ).

Apoptosis assay by flow cytometry

For analysis of cell proliferation, the dissociated tissue cells or cultured cells were re-suspended at a density of $10^{6}$ cells $/ \mathrm{ml}$ in PBS. After double staining with FITC-Annexin V and propidium iodide (PI) from a FITC Annexin V Apoptosis Detection Kit I (Becton-Dickinson Biosciences, San Jose, CA, USA), cells were analyzed using FACScan flow cytometer (Becton-Dickinson Biosciences) equipped with Cell Quest software (Becton-Dickinson Biosciences) for determination of Annexin V+ PI- apoptotic cells.

\section{Statistical analysis}

All data were statistically analyzed using one-way ANOVA with a Bonferroni correction, followed by Fisher's Exact Test for comparison of two groups (GraphPad Prism, GraphPad Software, Inc. La Jolla, CA, USA). Bivariate correlations were calculated by Spearman's Rank Correlation Coefficients. Kaplan-Meier curves were sued to analyze the patient survival by miR-429 levels. All values are depicted as mean \pm standard deviation and are considered significant if $\mathrm{p}<0.05$. 


\section{Results}

Decreased miR-429 and increased Bcl-2 levels correlate in GC specimens

Using 40 GC specimens, we detected significantly higher levels of Bcl-2 (Fig. 1A), and significantly lower levels of miR-429 in GC specimens (Fig. 1B), compared to the adjacent non-tumor gastric tissues (NT) from the same patient. To examine the relationship between miR-429 and Bcl-2, we examined the levels of them in these 40 GC specimens. A strong inverse correlation was detected between miR-429 and Bcl-2 (Fig. 1C, $\gamma=-0.76, p<0.0001$ ). These data suggest presence of a causal link between miR-429 and Bcl-2 in GC cells. Next, we investigated whether the levels of miR-429 may correlate with overall survival of GC patients. The 40 patients were followed-up for 5 years. The median value of all 40 cases was chosen as the cutoff point for separating miR-429-high cases $(n=20)$ from miR-429-low cases $(n=20)$. Kaplan-Meier curves were performed, showing that miR-429-low GC patients had a significantly shorter overall survival, compared to miR-429-high GC patients (Fig. 1D).

MiR-429 targets 3'-UTR of Bcl-2 mRNA to inhibit its translation in GC cells

Since our data suggest a relationship between miR-429 and Bcl-2 in GC cells, we checked whether miR-429 may target Bcl-2 mRNA to suppress its translation. Bioinformatics analyses

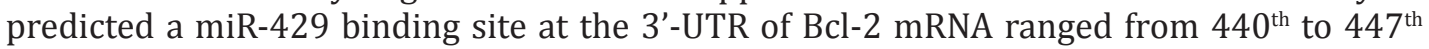

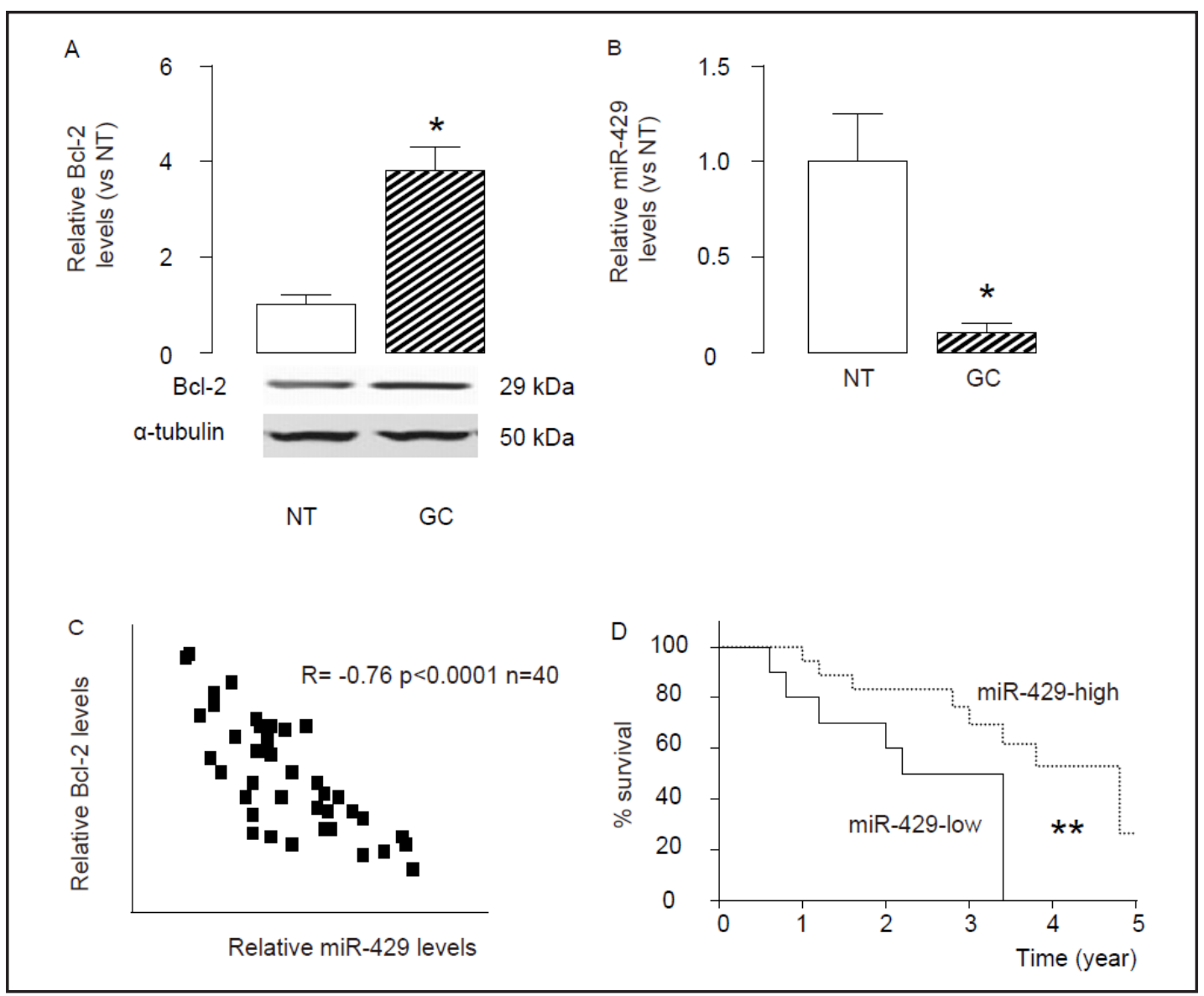

Fig. 1. Decreased miR-429 and correlated increased Bcl-2 are detected in GC. RT-qPCR on miR-429 and Western blot for Bcl-2 were performed on paired GC and the adjacent non-tumor gastric tissues (NT) from 40 patients. (A) Bcl-2 levels. (B) miR-429 levels. (C) A Correlation test between Bcl-2 and miR-429. (D) The 40 patients were followed-up for 5 years. The median value of all 40 cases was chosen as the cutoff point for separating miR-429-high cases $(n=20)$ from miR-429-low cases $(n=20)$. Kaplan-Meier curves were performed to evaluate the overall survival of the GC patients, based on miR-429 levels. ${ }^{*} \mathrm{p}<0.05$. $\mathrm{N}=40$. 
A

predicted binding of miR-429 to 3'-UTR on Bcl-2

5. AcGaggugugacuaAcuaugcaA 3'

Bcl-2 3'-UTR (440-447)

$3^{\prime}$

| || || || |

CUAGUGAAACACUGAUACGUU 5' miR-429

B
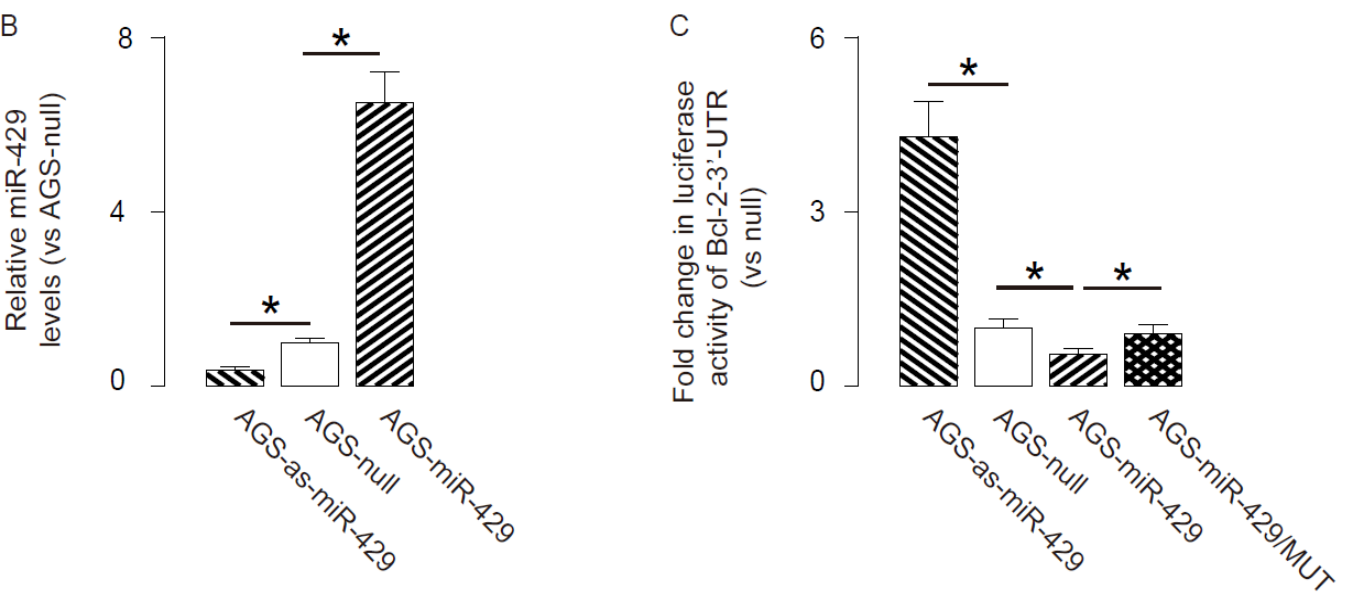

Fig. 2. MiR-429 targets 3'UTR of Bcl-2 mRNA to inhibit its expression. (A) Bioinformatics analyses showing binding of miR-429 to the 3'-UTR of Bcl-2 mRNA. (B) We either overexpressed miR-429, or inhibited miR429 in a human GC cell line, AGS, by transfection of the cells with a miR-429-expressing plasmid (AGSmiR-429), or with a plasmid carrying miR-429 antisense (AGS-as-miR-429). The AGS cells were also transfected with a plasmid carrying a null sequence as a control (AGS-null). The modification of miR-429 levels in AGS cells was confirmed by RT-qPCR. (C) MiR-429-modified AGS cells were transfected with $1 \mu \mathrm{g}$ of Bcl-2-3'UTR luciferase-reporter plasmid. The luciferase activities were examined. * $\mathrm{p}<0.05 . \mathrm{N}=5$.
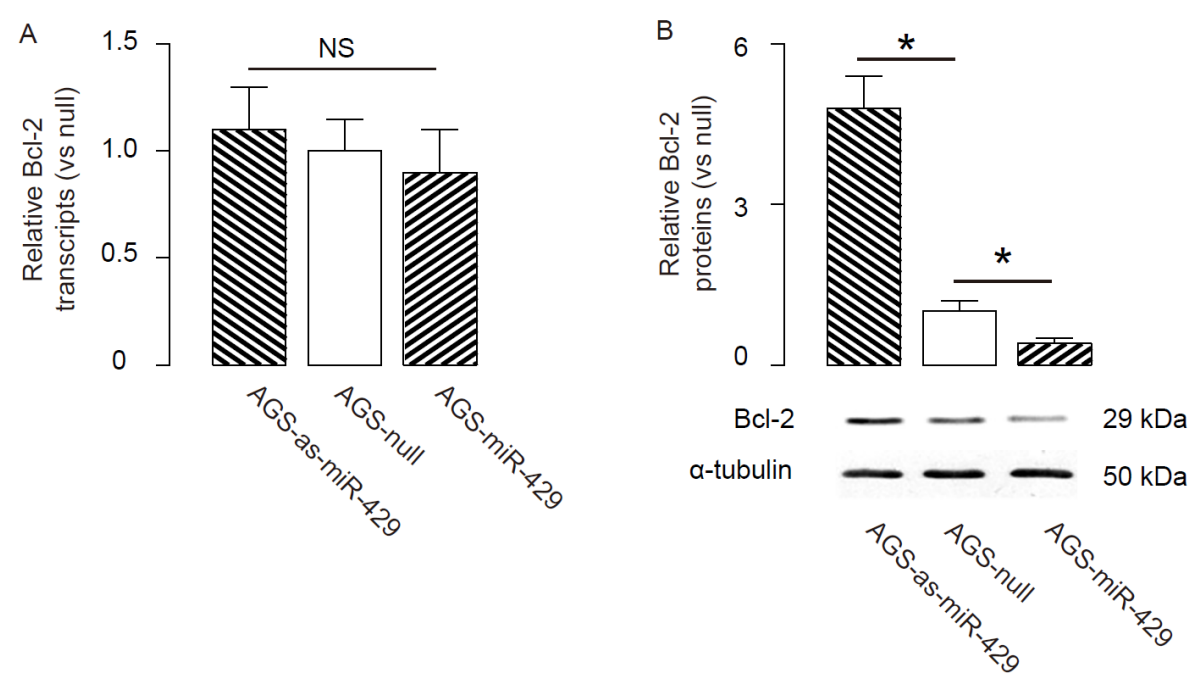

Fig. 3. MiR-429 decreases Bcl-2 protein, but not mRNA, in GC cells. (A-B) The Bcl-2 mRNA levels (A) and protein levels (B) in miR-429-modified AGS cells. *p<0.05. NS: non-significant. $N=5$.

base site (Fig. 2A). In order to figure out whether the binding of miR-429 to Bcl-2 mRNA in GC cells is functional, we either overexpressed miR-429, or inhibited miR-429 in a human GC cell line, AGS cells, by transfecting the cells with plasmids carrying either a miR-429- 


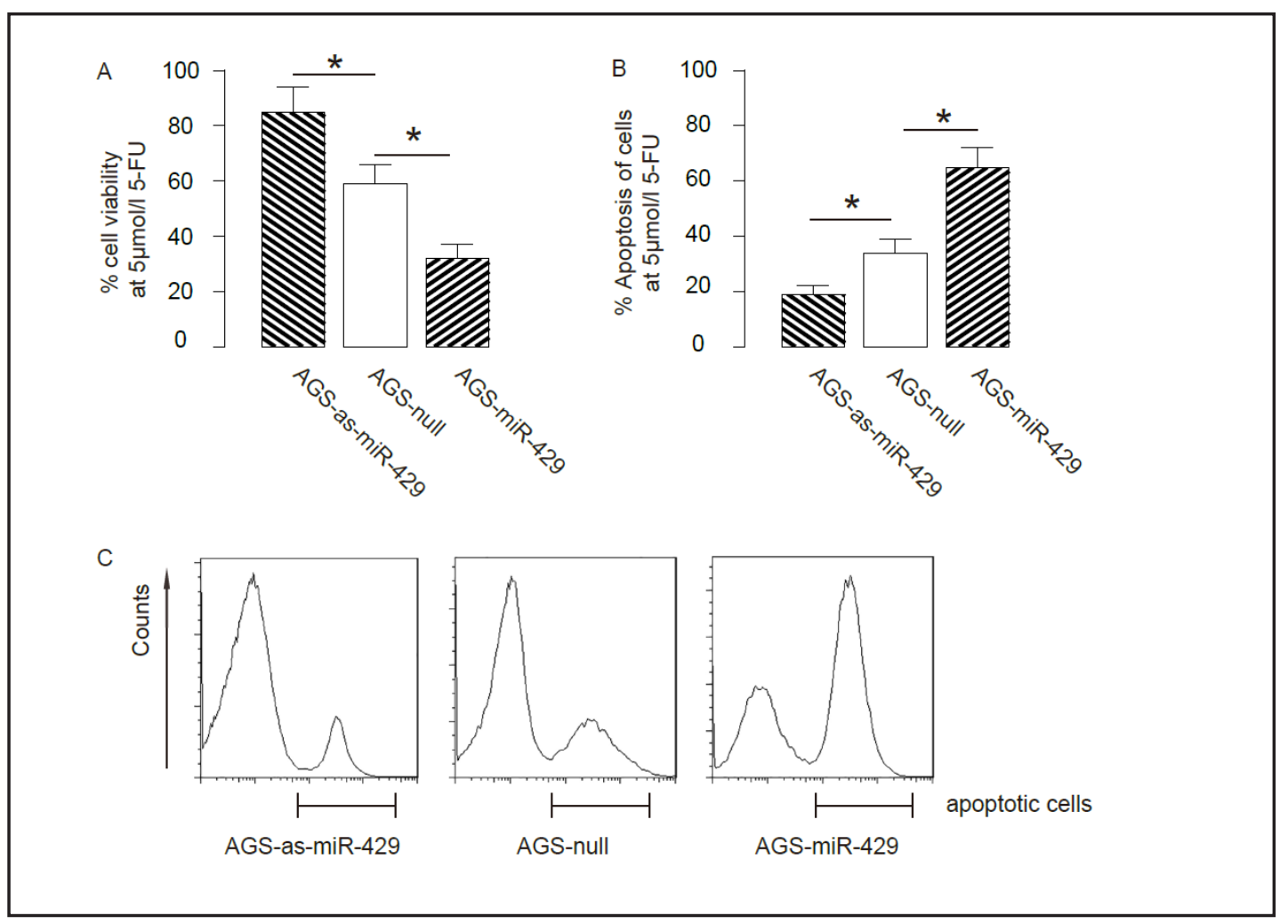

Fig. 4. MiR-429 increases 5-FU-induced GC cell apoptosis. (A) The effects of miR-429 modification on GC cell viability at presence of $5 \mu \mathrm{mol} / \mathrm{l} 5$-FU in an CCK-8 assay. (B) Fluorescence-based apoptosis assay on miR429-modified AGS cells, shown by quantification (B), and by representative flow charts (C). * $p<0.05$. N=5.

mimic (AGS-miR-429), or a miR-429 antisense (AGS-as-miR-429). The AGS cells were also transfected with a plasmid carrying null sequence as a control (AGS-null). The overexpression or inhibition of miR-429 in AGS cells was confirmed by RT-qPCR (Fig. 2B). MiR-429-modified AGS cells were then transfected with $1 \mu \mathrm{g}$ of Bcl-2 3'UTR luciferase-reporter plasmid or with $1 \mu \mathrm{g}$ of Bcl-2 $3^{\prime} \mathrm{UTR}$ luciferase-reporter plasmid with a site mutation between $440^{\text {th }}$ to $447^{\text {th }}$ base site. The luciferase activities were quantified in these cells, suggesting that the binding of miR-429 to 3'-UTR of Bcl-2 mRNA results in suppression of Bcl-2 protein translation (Fig. 2C).

MiR-429 reduces Bcl-2 protein, but not $m R N A$, in GC cells

We found that modification of miR-429 levels in AGS cells did not change mRNA levels of Bcl-2 (Fig. 3A). However, overexpression of miR-429 significantly decreased Bcl-2 protein levels while depletion of miR-429 significantly increased Bcl-2 protein levels in AGS cells, by Western blot (Fig. 3B). These data confirm that miR-429 inhibits translation of the Bcl-2 mRNA in GC cells.

MiR-429 increases 5-FU-induced GC cell apoptosis

Next, we examined the effects of miR-429 modification on GC cell viability at presence of 5-FU in a CCK-8 assay. We found that overexpression of miR-429 resulted in decreases in cell viability of AGS cells, and depletion of miR-429 resulted in increases in cell viability of AGS cells (Fig. 4A). Then we did fluorescence-based apoptosis assay, and found that overexpression of miR-429 resulted in increases in AGS cell apoptosis, and depletion of miR-429 resulted in decreases in AGS cell viability, shown by quantification (Fig. 4B), and by representative flow charts (Fig. 4C). Hence, miR-429 increases 5-FU-induced GC cell apoptosis. Together, our 
Fig. 5. Schematic model. MiR-429 suppression in GC promotes Bcl-2-mediated cancer cell survival against chemotherapy-induced cell death. Re-expression of miR-429 levels in GC cells may enhance cancer apoptosis during chemotherapy.

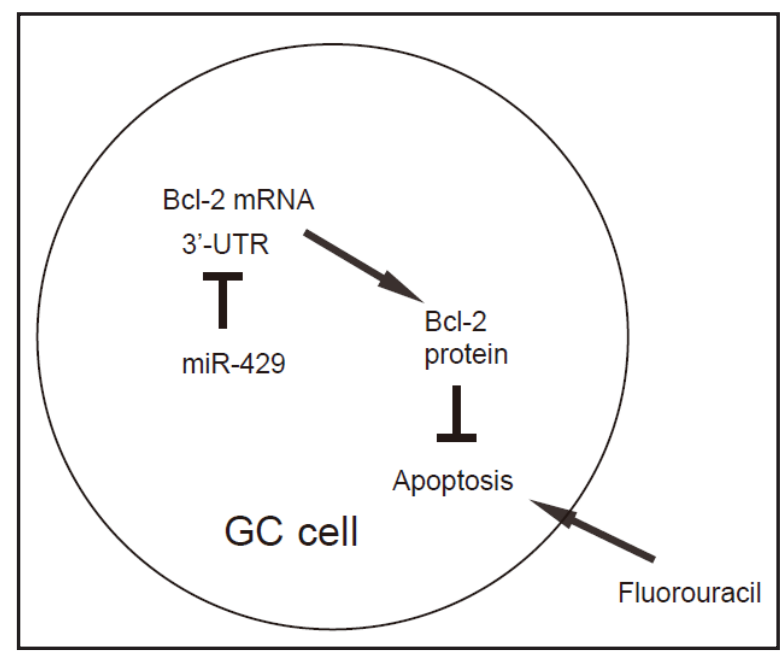

data suggest that miR-429 suppression in GC promotes Bcl-2-mediated cancer cell survival against chemotherapy-induced cell death. Re-expression of miR-429 levels in GC cells may enhance cancer apoptosis during chemotherapy (Fig. 5).

\section{Discussion}

Many miRNAs are essential in the processes including initiation, progression, invasion, metastases and resistance of chemotherapy of GC, which may either function by itself or in a cooperative manner with other key regulators to coordinate the pathogenesis of GC. Hence, understanding of the regulation of aberrantly expressed miRNAs in GC carcinogenesis may help to clarify the mechanisms underlying GC progression to improve the outcome of the therapy [22].

The resistance of GC cells to chemotherapy largely results from the activation of antiapoptotic machinery and suppression of apoptotic machinery inside the GC cells. From previous studies, we found that Bcl-2 activation might be a key responsive factor upon 5-FU treatment in GC cells. 5-FU induced activation of apoptosis-association proteins, e.g. Bid, Bak, Bad, resulting in upregulation of Cytochrome $\mathrm{C}$ and caspases to initiate the apoptotic process. Bcl-2 efficiently suppresses activation of these proteins, but the activation of Bcl-2 in response to chemotherapy may be case-dependent.

Here, by sequence matching using bioinformatics analyses, we found a few of candidate miRNAs that target Bcl-2, including miR-429, miR-30, miR-22, miR-25, miR-32, miR-92, miR-363, miR-367, miR-99, miR-27, miR-128, etc. Among all these miRNAs, we specifically detected a significant decrease in miR-429 in GC specimens, compared to paired non-tumor tissue. Hence, we hypothesized that miR-429 may target and regulate Bcl-2 in GC cells. Correlation test between miR-429 and Bcl-2 further supported this hypothesis, and luciferase reporter assay confirmed that miR-429 may bind Bcl-2 mRNA to impair its translation. Then, we modified miR-429 levels in GC cells, and found that it did not affect Bcl-2 mRNA, but altered the protein. These data were further strengthened the proposed mechanisms.

Moreover, miR-429-mediated changes in Bcl-2 seemed to result in changes in cell viability at presence of 5-FU. We have examined several other GC cell lines, e.g. SNU-5, and obtained essentially similar results. Thus, a possibility of the results to be cell-line-dependence could be excluded. The effects of miR-429 on cell invasion were not analyzed in the current study.

To summarize, we propose a model to explain the mechanisms underlying resistance of GC cells to chemotherapy. GC cells downregulate miR-429, resulting in enhanced Bcl-2 protein translation, which subsequently allows GC cells to survive upon chemotherapy. On the other hand, re-expression of miR-429 levels in GC cells may enhance cancer apoptosis during chemotherapy. 


\section{Cellular Physiology Cell Physiol Biochem 2015;37:1572-1580

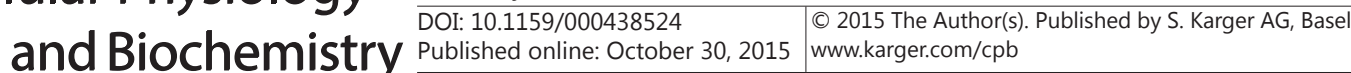 \\ Zhu et al.: MiR-429 Induces GC Cell Apoptosis via Bcl-2}

\section{Disclosure Statement}

The authors have declared that no competing interests exist.

\section{References}

1 Wu W, Ding H, Cao J, Zhang W: Fbxl5 inhibits metastasis of gastric cancer through suppressing snail1. Cell Physiol Biochem 2015;35:1764-1772.

2 Liu G, Jiang C, Li D, Wang R, Wang W: Mirna-34a inhibits egfr-signaling-dependent mmp7 activation in gastric cancer. Tumour Biol 2014;35:9801-9806.

3 Mao D, Zhang Y, Lu H, Zhang H: Molecular basis underlying inhibition of metastasis of gastric cancer by anti-vegfa treatment. Tumour Biol 2014;35:8217-8223.

4 Ye Y, Zhou X, Li X, Tang Y, Sun Y, Fang J: Inhibition of epidermal growth factor receptor signaling prohibits metastasis of gastric cancer via downregulation of mmp7 and mmp13. Tumour Biol 2014;35:1089110896.

5 Zhao Z, Han F, Yang S, Wu J, Zhan W: Oxamate-mediated inhibition of lactate dehydrogenase induces protective autophagy in gastric cancer cells: Involvement of the akt-mtor signaling pathway. Cancer Lett 2015;358:17-26.

6 Ge J, Chen Z, Huang J, Chen J, Yuan W, Deng Z, Chen Z: Upregulation of autophagy-related gene-5 (atg-5) is associated with chemoresistance in human gastric cancer. PLoS One 2014;9:e110293.

7 Hosogi S, Kusuzaki K, Inui T, Wang X, Marunaka Y: Cytosolic chloride ion is a key factor in lysosomal acidification and function of autophagy in human gastric cancer cell. J Cell Mol Med 2014;18:1124-1133.

8 Liu M, Li CM, Chen ZF, Ji R, Guo QH, Li Q Zhang HL, Zhou YN: Celecoxib regulates apoptosis and autophagy via the pi3k/akt signaling pathway in sgc-7901 gastric cancer cells. Int J Mol Med 2014;33:1451-1458.

9 Tang C, Yang L, Jiang X, Xu C, Wang M, Wang Q Zhou Z, Xiang Z, Cui H: Antibiotic drug tigecycline inhibited cell proliferation and induced autophagy in gastric cancer cells. Biochem Biophys Res Commun 2014;446:105-112.

10 Li DH, Pan ZK, Ye F, An HX, Wu JX: S-1-based versus 5-fu-based chemotherapy as first-line treatment in advanced gastric cancer: A meta-analysis of randomized controlled trials. Tumour Biol 2014;35:82018208.

11 Lu ZM, Luo TH, Nie MM, Fang GE, Ma LY, Xue XC, Wei G, Ke CW, Bi JW: Influence of ercc1 and ercc4 polymorphisms on response to prognosis in gastric cancer treated with folfox-based chemotherapy. Tumour Biol 2014;35:2941-2948.

12 Wu XJ, Yuan P, Li ZY, Bu ZD, Zhang LH, Wu AW, Zong XL, Li SX, Shan F, Ji X, Ren H, Ji JF: Cytoreductive surgery and hyperthermic intraperitoneal chemotherapy improves the survival of gastric cancer patients with ovarian metastasis and peritoneal dissemination. Tumour Biol 2013;34:463-469.

13 Shin D, Kwon HY, Sohn EJ, Nam MS, Kim JH, Lee JC, Ryu SY, Park B, Kim SH: Upregulation of death receptor 5 and production of reactive oxygen species mediate sensitization of pc-3 prostate cancer cells to trail induced apoptosis by vitisin a. Cell Physiol Biochem 2015;36:1151-1162.

14 Saleh AM, Aljada A, El-Abadelah MM, Sabri SS, Zahra JA, Nasr A, Aziz MA: The pyridone-annelated isoindigo (5'-cl) induces apoptosis, dysregulation of mitochondria and formation of ros in leukemic hl-60 cells. Cell Physiol Biochem 2015;35:1958-1974.

15 Yun M, Lee D, Park MN, Kim EO, Sohn EJ, Kwon BM, Kim SH: Cinnamaldehyde derivative (cb-pic) sensitizes chemo-resistant cancer cells to drug-induced apoptosis via suppression of mdr1 and its upstream stat3 and akt signalling. Cell Physiol Biochem 2015;35:1821-1830.

16 Xiong Y, Ye T, Wang M, Xia Y, Wang N, Song X, Wang F, Liu L, Zhu Y, Yang F, Wei Y, Yu L: A novel cinnamide ylt26 induces breast cancer cells apoptosis via ros-mitochondrial apoptotic pathway in vitro and inhibits lung metastasis in vivo. Cell Physiol Biochem 2014;34:1863-1876.

17 Li X, Huo X, Zhang C, Ma X, Han F, Wang G: Role of continuous high thoracic epidural anesthesia in hippocampal apoptosis after global cerebral ischemia in rats. Cell Physiol Biochem 2014;34:1227-1240.

18 Xie M, Yi X, Wang R, Wang L, He G, Zhu M, Qi C, Liu Y, Ye Y, Tan S, Tang A: 14-thienyl methylene matrine (yyj18), the derivative from matrine, induces apoptosis of human nasopharyngeal carcinoma cells by targeting mapk and pi3k/akt pathways in vitro. Cell Physiol Biochem 2014;33:1475-1483. 


\section{Cellular Physiology Cell Physiol Biochem 2015;37:1572-1580 \begin{tabular}{ll|l} 
DOI: 10.1159/000438524 & $\begin{array}{l}\text { O 2015 The Author(s). Published by S. Karger AG, Basel } \\
\text { www.karger.com/cpb }\end{array}$
\end{tabular} \\ Zhu et al.: MiR-429 Induces GC Cell Apoptosis via Bcl-2}

19 Zhu Y, Xia Y, Ye T, Shi X, Song X, Liu L, Zeng J, Wang N, Luo Y, Han Y, Yu L: A novel small-molecule ylt205 induces apoptosis in human colorectal cells via mitochondrial apoptosis pathway in vitro and inhibits tumor growth in vivo. Cell Physiol Biochem 2014;33:933-944.

20 Cui Y, Chen J, He Z, Xiao Y: Suz12 depletion suppresses the proliferation of gastric cancer cells. Cell Physiol Biochem 2013;31:778-784.

21 Mei Q, Li F, Quan H, Liu Y, Xu H: Busulfan inhibits growth of human osteosarcoma through mir-200 family micrornas in vitro and in vivo. Cancer Sci 2014;105:755-762.

22 Wang F, Xiao W, Sun J, Han D, Zhu Y: Mirna-181c inhibits egfr-signaling-dependent mmp9 activation via suppressing akt phosphorylation in glioblastoma. Tumour Biol 2014;35:8653-8658.

23 Di Leva G, Croce CM: Mirna profiling of cancer. Curr Opin Genet Dev 2013;23:3-11.

24 Pereira DM, Rodrigues PM, Borralho PM, Rodrigues CM: Delivering the promise of mirna cancer therapeutics. Drug Discov Today 2013;18:282-289.

25 Zhou X, Xia Y, Su J, Zhang G: Down-regulation of mir-141 induced by helicobacter pylori promotes the invasion of gastric cancer by targeting stat4. Cell Physiol Biochem 2014;33:1003-1012.

26 Coronnello C, Benos PV: Comir: Combinatorial microrna target prediction tool. Nucleic Acids Res 2013;41:W159-164. 